\title{
A DIRECT PROOF OF GLOBAL EXISTENCE FOR THE DIRAC-KLEIN-GORDON EQUATIONS IN ONE SPACE DIMENSION
}

\author{
Yung-fu Fang
}

\begin{abstract}
We establish local and global existence results for Dirac-KleinGordon equations in one space dimension, employing a null form estimate and a fixed point argument.
\end{abstract}

\section{InTRODUCTION AND Main Results}

In the present work, we want to consider the Cauchy problem for the DiracKlein-Gordon equations

$$
\left\{\begin{array}{l}
\mathcal{D} \psi=\phi \psi ; \quad(t, x) \in \mathbb{R} \times \mathbb{R}^{1}, \\
\square \phi=\bar{\psi} \psi ; \\
\psi(0)=\psi_{0}, \quad \phi(0)=\phi_{0}, \quad \partial_{t} \phi(0)=\phi_{1},
\end{array}\right.
$$

where $\psi$ is a 2-spinor field takes value in $\mathbb{C}^{4}, \phi$ is a scalar field takes value in $\mathbb{R}$, $\mathcal{D}$ is the Dirac operator, $\mathcal{D}=-i \gamma^{\mu} \partial_{\mu}, \mu=0,1$, and $\gamma^{\mu}$ are the Dirac matrices, which can be written as follows. First let us define the $2 \times 2$ matrix $\sigma_{1}$,

$$
\sigma_{1}=\left(\begin{array}{ll}
0 & 1 \\
1 & 0
\end{array}\right)
$$

The matrices $\gamma^{\mu}$ are defined by

$$
\gamma^{0}=\left(\begin{array}{cc}
I_{2} & 0 \\
0 & -I_{2}
\end{array}\right), \quad \gamma^{1}=\left(\begin{array}{cc}
0 & \sigma_{1} \\
-\sigma_{1} & 0
\end{array}\right)
$$

Received July 31, 2002; revised December 19, 2002.

Communicated by S. B. Hsu.

2000 Mathematics Subject Classification: 35L05.

Key words and phrases: Null form estimate.

Partially supported by National Science Council at Taiwan, NSC90-2115-M006-025. 
where $I_{2}$ is the $2 \times 2$ identity matrix, the wave operator $\square=-\partial_{t t}+\partial_{x x}$, and $\bar{\psi}=\psi^{\dagger} \gamma^{0}$, where $\dagger$ is the complex conjugate transpose.

The purpose of this work is to demonstrate a new proof of null form estimate, see [1], by employing a solution representation of DKG equations, which apparently is more simple. In a way, we give an interpretation of the null form structure depicted within the nonlinear term $\bar{\psi} \psi$, and it is different from that in [1]. This term has been observed for possessing the null form structure, see[1] and $[5,6]$.

The energy for the DKG problem is not positive definite, therefore it is not applicable. However we have the law of conservation of charge,

$$
\int|\psi(t)|^{2} d x=\text { constant }
$$

which can be applied to derive the global existence result, once we establish the local existence result. In this paper, the "charge class" solutions mean solutions with $\psi(t, \cdot) \in L^{2}(\mathbb{R})$.

In '73, Chadam showed that the Cauchy problem for the DKG equations has a global unique solution if $\psi_{0} \in H^{1}, \phi_{0} \in H^{1}, \phi_{1} \in L^{2}$, see [2]. In '93, Zheng proved that there exists a global weak solution to the Cauchy problem of the modified DKG equations, based on the technique of compensated compactness, with $\psi_{0} \in L^{2}$, $\phi_{0} \in H^{1}, \phi_{1} \in L^{2}$, see [8]. In '00, Bournaveas derived a new proof of a global existence for the DKG equations, based on a null form estimate, if $\psi_{0} \in L^{2}$, $\phi_{0} \in H^{1}, \phi_{1} \in L^{2}$, see [1].

The approach we adopt in this work is as follows: First, we will derive the solution representation for the Dirac equation, while the solution representation for the wave equation is well known. Next, we will use it to estimate the quadratic term $\bar{\psi} \psi$. In addition to this, the derivations of some necessary estimates become straight forward. Finally we can prove the local and global existence results of DKG equations, which are parallel to those in [1] and [8].

The main result in this work is as follows.

Theorem 1. (Global Existence) If the initial data of (1), $\psi_{0} \in L^{2}, \phi_{0} \in H^{1}$, $\phi_{1} \in L^{2}$, then there is a unique global solution for (1).

\section{Solution RPRESEntation}

Recall that, for the wave equation,

$$
\left\{\begin{array}{l}
\square \phi=F, \quad(t, x) \in \mathbb{R}^{1} \times \mathbb{R}^{1}, \\
\phi(0)=\phi_{0}, \phi_{t}(0)=\phi_{1},
\end{array}\right.
$$


the solution representations is as follows:

(6)

$$
2 \phi(t, x)=\left[\phi_{0}(x+t)+\phi_{0}(x-t)\right]+\int_{x-t}^{x+t} \phi_{1}(y) d y+\int_{0}^{t} \int_{x-t+s}^{x+t-s} F(s, y) d y d s .
$$

Consider the Dirac equation,

$$
\left\{\begin{array}{l}
\mathcal{D} \psi=G ; \quad(t, x) \in \mathbb{R}^{1} \times \mathbb{R}^{1}, \\
\psi(0)=\psi_{0} .
\end{array}\right.
$$

First we take $\mathcal{D}$ on the Dirac equation, then (7) becomes

$$
\left\{\begin{array}{l}
\square \psi=\mathcal{D} G ; \quad(t, x) \in \mathbb{R}^{1} \times \mathbb{R}^{1}, \\
\psi(0)=\psi_{0}, \quad \partial_{t} \psi(0)=-\gamma^{0} \gamma^{1} \frac{d}{d x} \psi_{0} .
\end{array}\right.
$$

Applying the formula (6) and simplifying the expression, we finally have

(9)

$$
\begin{aligned}
2 \psi(t, x)= & \left(\gamma^{0}+\gamma^{1}\right) \gamma^{0} \psi_{0}(x+t)+\left(\gamma^{0}-\gamma^{1}\right) \gamma^{0} \psi_{0}(x-t)+ \\
& i \int_{0}^{t}\left(\gamma^{0}+\gamma^{1}\right) G(s, x+t-s) d s+i \int_{0}^{t}\left(\gamma^{0}-\gamma^{1}\right) G(s, x-t+s) d s .
\end{aligned}
$$

The formula (9) can also be derived in the approach via Fourier transform. First we take the Fourier transform on the Dirac equation (9) over the space variable. Then we solve the resulting ODE to get a formula for $\hat{\psi}(t, \xi)$. Finally we take the inverse Fourier transform to obtain a solution formula for $\psi(t, x)$. We will not present the derivation of (9) via Fourier transform approach, due to the fact that it is straight forward.

\section{ESTIMATE}

Lemma 1. For the solution of the Dirac equation (7), we have

$$
\|\psi(t)\|_{L^{2}} \cdot C\left(\left\|\psi_{0}\right\|_{L^{2}}+\int_{0}^{T}\|G(s)\|_{L^{2}} d s\right) .
$$

This can be shown directly. 
Consider two Dirac equations,

$$
\left\{\begin{array}{l}
\mathcal{D} \psi_{j}=G_{j}, \quad j=1,2, \\
\psi_{j}(0)=\psi_{0 j} .
\end{array}\right.
$$

Lemma 2. (Null Form Estimate)

$$
\begin{aligned}
&\left\|\bar{\psi}_{1} \psi_{2}\right\|_{L^{2}\left([0, T), L^{2}\right)} \\
& \cdot C\left(\left\|\psi_{01}\right\|_{L^{2}}+\int_{0}^{T}\left\|G_{1}(s)\right\|_{L^{2}} d s\right)\left(\left\|\psi_{02}\right\|_{L^{2}}+\int_{0}^{T}\left\|G_{2}(s)\right\|_{L^{2}} d s\right) .
\end{aligned}
$$

Proof. For simplicity, we demonstrate a special case when $\psi_{1}=\psi_{2}$, and the general case will follow. Consider the Dirac equation (7) and write its solution as

$$
2 \psi(t, x)=U_{+}+U_{-}+i V_{+}+i V_{-},
$$

where

$$
\begin{gathered}
U_{ \pm}(t, x)=\left(\gamma^{0} \pm \gamma^{1}\right) \gamma^{0} \psi_{0}(x \pm t) \\
V_{ \pm}(t, x)=\int_{0}^{t}\left(\gamma^{0} \pm \gamma^{1}\right) G(s, x \pm(t-s)) d s
\end{gathered}
$$

Throughout calculations, we get

$$
\begin{gathered}
\bar{U}_{+} U_{+}=\bar{U}_{-} U_{-}=\bar{V}_{+} V_{+}=\bar{V}_{-} V_{-}=0, \\
\bar{U}_{+} V_{+}=\bar{U}_{-} V_{-}=\bar{V}_{+} U_{+}=\bar{V}_{-} U_{-}=0, \\
\left\|\bar{U}_{+} U_{-}\right\|_{L^{2}\left([0, T), L^{2}\right)} \cdot C\left\|\psi_{0}\right\|_{L^{2}}^{2},
\end{gathered}
$$

$$
\left\|\bar{V}_{+} U_{-}\right\|_{L^{2}\left([0, T), L^{2}\right)} \cdot C\left\|\psi_{0}\right\|_{L^{2}} \int_{0}^{T}\|G(s)\|_{L^{2}} d s,
$$

$$
\left\|\bar{U}_{+} V_{-}\right\|_{L^{2}\left([0, T), L^{2}\right)} \cdot C\left\|\psi_{0}\right\|_{L^{2}} \int_{0}^{T}\|G(s)\|_{L^{2}} d s,
$$

$$
\left\|\bar{V}_{+} V_{-}\right\|_{L^{2}\left([0, T), L^{2}\right)} \cdot C\left(\int_{0}^{T}\|G(s)\|_{L^{2}} d s\right)^{2} .
$$

The rest cases are analogous. Among these cases, we only demonstrate the case of $\bar{V}_{+} V_{-}$. Since

$$
\bar{V}_{+} V_{-}=2 \int_{0}^{t} \int_{0}^{t} G^{\dagger}(s, x+t-s)\left(\gamma^{0}-\gamma^{1}\right) G(r, x-t+r) d r d s
$$


hence we have

(23)

$$
\left\|\bar{V}_{+} V_{-}\right\|_{L^{2}\left([0, T), L^{2}\right)}
$$

- $C\left(\int_{0}^{T} \int\left(\int_{0}^{t} \int_{0}^{t}\left|G^{\dagger}(s, x+t-s)\left(\gamma^{0}-\gamma^{1}\right) G(r, x-t+r)\right| d r d s\right)^{2} d x d t\right)^{\frac{1}{2}}$

- $C \int_{0}^{T} \int_{0}^{T}\left(\int_{0}^{T} \int\left|G^{\dagger}(s, x+t-s)\right|^{2}|G(r, x-t+r)|^{2} d x d t\right)^{\frac{1}{2}} d r d s$

- $\quad C \int_{0}^{T} \int_{0}^{T}\|G(s)\|_{L^{2}}\|G(r)\|_{L^{2}} d r d s$

- $C\left(\int_{0}^{T}\|G(s)\|_{L^{2}} d s\right)^{2}$.

This completes the proof of the lemma.

Lemma 3. For the wave equation (10), we have the energy estimate

(25) $\|\phi(t)\|_{H^{1}}+\left\|\phi_{t}(t)\right\|_{L^{2}} \cdot C(T)\left(\left\|\phi_{0}\right\|_{H^{1}}+\left\|\phi_{1}\right\|_{L^{2}}+\int_{0}^{T}\|F(s)\|_{L^{2}} d s\right)$.

This can be derived directly.

\section{EXISTENCE}

Let $(\psi, \phi)$ and $\left(\psi^{\prime}, \phi^{\prime}\right)$ be two charge class solutions of DKG equations. We define the following quantities:

$$
\begin{gathered}
J(0)=\left\|\psi_{0}\right\|_{L^{2}}+\left\|\phi_{0}\right\|_{H^{1}}+\left\|\phi_{1}\right\|_{L^{2}} ; \\
J^{\prime}(0)=\left\|\psi_{0}^{\prime}\right\|_{L^{2}}+\left\|\phi_{0}^{\prime}\right\|_{H^{1}}+\left\|\phi_{1}^{\prime}\right\|_{L^{2}} ; \\
J(T)=\sup _{[0, T]}\left(\|\psi(t)\|_{L^{2}}+\|\phi(t)\|_{H^{1}}+\|\phi(t)\|_{L^{2}}\right) ; \\
J^{\prime}(T)=\sup _{[0, T]}\left(\left\|\psi^{\prime}(t)\right\|_{L^{2}}+\left\|\phi^{\prime}(t)\right\|_{H^{1}}+\left\|\phi^{\prime}(t)\right\|_{L^{2}}\right) ; \\
\Delta(0)=\left\|\psi_{0}-\psi_{0}^{\prime}\right\|_{L^{2}}+\left\|\phi_{0}-\phi_{0}^{\prime}\right\|_{H^{1}}+\left\|\phi_{1}-\phi_{1}^{\prime}\right\|_{L^{2}} ; \\
\Delta(T)=\sup _{[0, T]}\left(\left\|\psi(t)-\psi^{\prime}(t)\right\|_{L^{2}}+\left\|\phi(t)-\phi^{\prime}(t)\right\|_{H^{1}}+\left\|\phi_{t}(t)-\phi_{t}^{\prime}(t)\right\|_{L^{2}}\right) .
\end{gathered}
$$


Lemma 4. For the solution of the DKG equations (1), we have

$$
\|\phi(t)\|_{L^{\infty}(\mathbb{R})} \cdot C(T, J(0)) .
$$

This can be obtained by using the solution representation for $\phi$ and the law of conservation of charge, see [1].

Lemma 5. Let $T>0$ and let $(\psi, \phi)$ be a charge class solution of (1). Then there exists a constant $C>0$, depending only on $T$ and $J(0)$, such that

$$
J(T) \cdot C(T, J(0))
$$

Proof. Since $\|\psi(t)\|_{L^{2}}=\left\|\psi_{0}\right\|_{L^{2}}$, this takes care the first term of $J(T)$. For the terms involving $\phi$, we first compute

$$
\begin{aligned}
& \int_{0}^{T}\|F(s)\|_{L^{2}} d s=\int_{0}^{T}\|\bar{\psi} \psi(s)\|_{L^{2}} d s \\
& \text { - } T^{\frac{1}{2}}\|\bar{\psi} \psi\|_{L^{2}\left([0, T], L^{2}\right)} \\
& T^{\frac{1}{2}}\left(\left\|\psi_{0}\right\|_{L^{2}}+\int_{0}^{T}\|\mathcal{D} \psi(s)\|_{L^{2}} d s\right)^{2} \\
& \text { - } T^{\frac{1}{2}}\left(J(0)+\int_{0}^{T}\|\phi(s)\|_{L^{\infty}}\|\psi(s)\|_{L^{2}} d s\right)^{2} \\
& \text { - } C(T, J(0)) T^{\frac{1}{2}} \text {. }
\end{aligned}
$$

Now we apply the energy estimate to get

$$
\begin{array}{ll}
\|\phi(t)\|_{H^{1}}+\left\|\phi_{t}(t)\right\|_{L^{2}} \cdot & C(T)\left(J(0)+\int_{0}^{T}\|F(s)\|_{L^{2}} d s\right) \\
\cdot & C(T, J(0)) .
\end{array}
$$

This completes the proof of the lemma.

Lemma 6. Let $T>0$ and $(\psi, \phi),\left(\psi^{\prime}, \phi^{\prime}\right)$ be two charge class solutions of (1). Then there exists $\epsilon>0, C>0$, depending only on $J(0)$ and $J^{\prime}(0)$ such that if $T<\epsilon$, then

$$
\Delta(T) \cdot C \Delta(0) .
$$

Proof. Consider the difference of the two solutions, we have the following equations: 


$$
\left\{\begin{array}{l}
\mathcal{D}\left(\psi-\psi^{\prime}\right)=\left(\phi-\phi^{\prime}\right) \psi+\phi^{\prime}\left(\psi-\psi^{\prime}\right), \\
\square\left(\phi-\phi^{\prime}\right)=\overline{\left(\psi-\psi^{\prime}\right)} \psi+\overline{\psi^{\prime}}\left(\psi-\psi^{\prime}\right) .
\end{array}\right.
$$

For the first term of $\Delta(T)$, first we compute

$$
\begin{aligned}
& \left\|\mathcal{D}\left(\psi-\psi^{\prime}\right)(s)\right\|_{L^{2}} \\
\text {. } & \left\|\phi(s)-\phi^{\prime}(s)\right\|_{L^{\infty}}\|\psi(s)\|_{L^{2}}+\left\|\phi^{\prime}(s)\right\|_{L^{\infty}}\left\|\psi(s)-\psi^{\prime}(s)\right\|_{L^{2}} \\
\text { - } & \left\|\phi(s)-\phi^{\prime}(s)\right\|_{H^{1}} J(0)+C\left(T, J^{\prime}(0)\right)\left\|\psi(s)-\psi^{\prime}(s)\right\|_{L^{2}} \\
\text {. } & C\left(T, J(0), J^{\prime}(0)\right) \Delta(T) .
\end{aligned}
$$

Invoke (13), we have

$$
\begin{aligned}
\left\|\psi(t)-\psi^{\prime}(t)\right\|_{L^{2}} \cdot & C\left(\left\|\psi_{0}-\psi_{0}^{\prime}\right\|_{L^{2}}+\int_{0}^{T}\left\|\mathcal{D}\left(\psi-\psi^{\prime}\right)(s)\right\|_{L^{2}} d s\right) \\
\cdot & C\left(\Delta(0)+C\left(T, J(0), J^{\prime}(0)\right) T \Delta(T)\right) .
\end{aligned}
$$

For the other two terms, first we calculate

$$
\begin{aligned}
& \int_{0}^{T}\left\|\square\left(\phi-\phi^{\prime}\right)(s)\right\|_{L^{2}} d s \\
\cdot & \int_{0}^{T}\left\|\overline{\left(\psi-\psi^{\prime}\right)} \psi(s)\right\|_{L^{2}}+\left\|\overline{\psi^{\prime}}\left(\psi-\psi^{\prime}\right)(s)\right\|_{L^{2}} d s \\
\cdot & T^{\frac{1}{2}}\left(\left\|\overline{\left(\psi-\psi^{\prime}\right)} \psi\right\|_{L^{2}}+\left\|\overline{\psi^{\prime}}\left(\psi-\psi^{\prime}\right)\right\|_{L^{2}}\right),
\end{aligned}
$$

then we calculate, invoke (15),

$$
\left\|\overline{\left(\psi-\psi^{\prime}\right)} \psi\right\|_{L^{2}}
$$

$$
\begin{aligned}
& \text {. } C\left(\left\|\psi_{0}-\psi_{0}^{\prime}\right\|_{L^{2}}+\int_{0}^{T}\left\|\mathcal{D}\left(\psi-\psi^{\prime}\right)(s)\right\|_{L^{2}} d s\right)\left(\left\|\psi_{0}\right\|_{L^{2}}+\int_{0}^{T}\|\mathcal{D} \psi(s)\|_{L^{2}} d s\right) \\
& \text {. } C\left(T, J(0), J^{\prime}(0)\right)(\Delta(0)+T \Delta(T)) .
\end{aligned}
$$

The calculation for $\left\|\overline{\psi^{\prime}}\left(\psi-\psi^{\prime}\right)\right\|_{L^{2}}$ is the same. Therefore, applying (25), we get

$$
\begin{array}{ll} 
& \left\|\phi(t)-\phi^{\prime}(t)\right\|_{H^{1}}+\left\|\phi_{t}(t)-\phi_{t}^{\prime}(t)\right\|_{L^{2}} \\
\cdot & C(T)\left(\left\|\phi_{0}-\phi_{0}^{\prime}\right\|_{H^{1}}+\left\|\phi_{1}-\phi_{1}^{\prime}\right\|_{L^{2}}+\int_{0}^{T}\left\|\square\left(\phi-\phi^{\prime}\right)(s)\right\|_{L^{2}} d s\right) \\
\cdot & C(T)\left(\Delta(0)+T^{\frac{1}{2}} C\left(T, J(0), J^{\prime}(0)\right)\right)(\Delta(0)+T \Delta(T)) \\
\cdot & C\left(T, J(0), J^{\prime}(0)\right)(\Delta(0)+T \Delta(T)) .
\end{array}
$$


Now we assume $T \cdot 1$, thus

$$
C\left(T, J(0), J^{\prime}(0)\right) \cdot C\left(J(0), J^{\prime}(0)\right)=C\left(1, J(0), J^{\prime}(0)\right)
$$

and

$$
\Delta(T) \cdot C\left(J(0), J^{\prime}(0)\right)(\Delta(0)+T \Delta(T)) .
$$

This concludes the proof.

Combining (28), (31) and an iteration scheme, we have the following local existence result.

Theorem 2. (Local Existence) Let $\psi_{0} \in L^{2}(\mathbb{R}), \phi_{0} \in H^{1}(\mathbb{R})$, and $\phi_{1} \in$ $L^{2}(\mathbb{R})$. Then there exists a $T>0$, depending only on $J(0)$, and a unique charge class solution of (1) defined on $[0, T) \times \mathbb{R}$.

From Lemma 5, we have the inequality,

$$
J(T) \cdot C(T, J(0)),
$$

which ensures that we can always extend the solution beyond $T$. Thus we have a unique global solution for the Cauchy problem. The idea of this type of argument is originated by Segal, see [7].

\section{REMARK}

Using the solution representation in Fourier transform, we can take full advantage of the null form structure and derive the null form estimate,

$$
\|\bar{\psi} \psi\|_{L^{2}} \cdot C\left(\left\|\psi_{0}\right\|_{L^{2}}+\left\|\frac{\hat{\psi} G}{\hat{\psi} W^{\frac{1}{2}-\epsilon}}\right\|_{L^{2}}\right)^{2}
$$

where $\hat{\psi} W=|| \tau|-| \xi||+1$. Equipping with this estimate, together with other estimates, the result of global existence can be improved, i.e. if $\psi_{0} \in L^{2}(\mathbb{R})$, $\phi_{0} \in H^{\frac{1}{2}}(\mathbb{R})$, and $\phi_{1} \in H^{-\frac{1}{2}}(\mathbb{R})$, then we have a unique global solution. The proof will appear elsewhere.

\section{ACKNOWLEDGEMENT}

The author wants to express his gratitude to M. Grillakis for his encouragement and inspiring conversation, and also to Tai-ping Liu for his helps when I visited Sinica in January 2002. 


\title{
REFERENCES
}

1. N. Bournaveas, A New Proof of Global Existence for the Dirac Klein-Gordon Equations in One Space Dimension, J. Funct. Anal. 173 (2000), 203-213.

2. J. M. Chadam, Global solutions of the Cauchy problem for the (classical) MaxwellDirac equations in one space dimension, J. Funct. Anal. 13 (1973), 173-184.

3. J. M. Chadam and R. T. Glassey, Properties of the solutions of the Cauchy problem for the (classical) coupled Maxwell-Dirac equations in one space dimension, em Proc. Amer. Math. Soc. 43 (1974), 373-378.

4. - On Certain Global Solutions of the Cauchy Problem for the (Classical) Coupled Klein-Gordon-Dirac Equations in One and Three Space Dimensions, Arch. Rational Mech. Anal. 54 (1974), 223-237.

5. S. Klainerman and M. Machedon, Space-time estimates for the null form and the local existence theorem, em Comm. Pure Appl. Math. 46 (1993), 1221-1268.

6. - On the Maxwell-Klein-Gordon equations with finite energy em Duke Math. J. 74 (1994), 19-44.

7. I. E. Segal, Non-linear semigroups, Ann. of Math. 78 (1963), 339-364.

8. Y. Zhang, Regularity of weak Solution to a Two-Dimensional Modified Dirac-KleinGordon System of Equations Commun. Math. Phys. 151 (1993), 67-87.

\author{
Yung-fu Fang \\ Department of Mathematics, \\ National Cheng Kung University, \\ Tainan 701, Taiwan. \\ E-mail: fang@math.ncku.edu.tw
}

